\title{
How does age affect the relationship between weight and health utility during the middle years of childhood?
}

\author{
Katie Eminson ${ }^{1} \cdot$ Alastair Canaway $^{2}$ (D) $\cdot$ Peymané Adab $^{3} \cdot$ Emma Lancashire $^{3} \cdot$ Miranda Pallan $^{3} \cdot$ Emma Frew $^{4}$ D
}

Accepted: 20 January 2018 / Published online: 5 February 2018

(c) The Author(s) 2018. This article is an open access publication

\begin{abstract}
Purpose The limited literature examining weight status and preference-based health-related quality of life (HRQL) in young children is equivocal. This study aims to examine how the association between weight status and preference-based HRQL changes as children develop between the ages of 6 and 10 years old.

Methods The Child Health Utility 9D (CHU-9D) was used to determine preference-based HRQL. Height and weight data were also collected and used to calculate z-BMI adjusted for age and gender. 1467 children were recruited from 54 schools across the West Midlands. Data were collected at four time points over 5 years. Impact of weight on dimensions of HRQL was assessed via the distribution of responses to CHU-9D dimensions by weight status. Multi-level regression analysis controlling for ethnicity, deprivation and other relevant co-variates was conducted to examine the relationship between weight and HRQL. Results There was no evidence to suggest that the weight status impacted upon the distribution of responses to CHU-9D dimensions. Correspondingly, the multi-level regression analysis found no statistically significant differences in CHU-9D scores between underweight, healthy weight, overweight and obese children.

Conclusions The evidence surrounding the link between preference-based HRQL and weight status in children is limited. This study found no association between weight status and HRQL as measured by the CHU-9D in children between the ages of 5 and 10 years in the UK. Given this, it is recommended that future studies aiming to prevent obesity in children in their middle years do not rely solely on preference-based measures for economic evaluation, and instead focus on capturing clinical or wellbeing outcomes.
\end{abstract}

Keywords Health-related quality of life $\cdot$ Child health $\cdot$ Obesity $\cdot$ CHU-9D $\cdot$ Utility $\cdot$ Child weight status

\section{Background}

Economic evaluation acts as an aid to facilitate decisionmaking by providing information on the relative costs and effects of competing interventions [1]. A common application is to measure costs from a health service/social care

Electronic supplementary material The online version of this article (https://doi.org/10.1007/s11136-018-1790-y) contains supplementary material, which is available to authorized users.

Alastair Canaway

a.canaway@warwick.ac.uk

1 UK Centre for Tobacco and Alcohol Studies, University of Nottingham, Nottingham NG7 2QL, UK

2 Warwick Clinical Trials Unit, University of Warwick, Coventry CV4 7AL, UK

3 Public Health, University of Birmingham, Public Health Building, Birmingham B15 2TT, UK

4 Health Economics Unit, University of Birmingham, Public Health Building, Birmingham B15 2TT, UK perspective and to measure intervention effects using a metric termed quality-adjusted life years (QALYs) [1]. The advantage of measuring all outcomes using QALYs is that it facilitates comparisons of cost-effectiveness across interventions in multiple disease and clinical contexts [2]. QALYs provide a measure that encompasses both quantity and quality of life and for the same level of resource use, preference will be given to interventions that yield the greatest number of QALYs. Unlike general quality of life measures, a special characteristic of QALYs is that the quality of life scores (utilities) used to calculate QALYs are preference based [3]. 
There is growing consensus that early intervention is key to the prevention of childhood obesity [4]. However, in a world of scarce resources and limited public health budgets, it is also important to demonstrate cost-effectiveness using commonly applied outcome measures such as QALYs. The QALY metric rests on the assumption that, in comparison with full health, being in a 'disease state' (obesity) yields a lower quality of life and thus any gains from intervening and avoiding that health state will be realised in QALY gains over time. Understanding the relationship between health utility and childhood obesity is therefore very important for economic evaluation and resultant decision-making.

Of the few studies that have explored this, Frew et al. [5] found no evidence of a negative relationship between health utility and weight status in children aged 5-6 years or in children aged 6-7 years [6]. Both studies used the CHU-9D. Likewise, Belfort et al. [7] found no negative relationships in children and adolescents aged 5-18 years using the Health Utility Index-Mark 3. In contrast, however, a recent Australian study using the CHU-9D [8] did find a significant negative relationship between weight status and health utility in children aged 10 years, and Boyle et al. (2010) [9] found a significant negative relationship in 11-15-year-old children using the EQ-5D and a non-preference-based instrument, the PedsQL. The evidence for understanding this relationship is therefore inconsistent. Furthermore, to date, studies have been cross-sectional in nature. No study has followed the same cohort of children over time to examine whether the relationship changes as children develop and mature through their 'middle years'. The middle years (age 6-12) is an important time to focus on, as it is particularly apt for childhood obesity prevention [10].

This study aimed to assess health utility in children followed up from age 5 to 10 years to examine how weight affects health utility as a child develops, and to evaluate the implications of this for resource allocation decision-making.

\section{Methods}

\section{Data collection methods}

The data within this study were collected as part of the West Midlands ActiVe lifestyle and healthy Eating in School children (WAVES) study. The WAVES study was a cluster-randomised, controlled trial based in the West Midlands, UK, which assessed the clinical effectiveness and cost-effectiveness of an obesity prevention intervention programme targeting young children. The study was funded by the National Institute for Health Research (ISRCTN97000586) and took place between 2010 and 2015 having obtained full ethics approval (NHS REC No. 10/H1202/69). The WAVES study was conducted in 54 primary schools spread across the West
Midlands region with randomisation occurring at the school level. Data were collected at both the cluster (school) and within-cluster (individual pupils and their parents) levels [11].

A weighted random sampling process was used to select 54 schools from among 980 that were eligible. Sampling took account of the ethnic mix of pupils and was weighted to increase the likelihood of selecting schools that had a higher minority ethnic population with a 3:1 ratio to ensure that there would be sufficient power to detect differences across ethnicities [11]. All year 1 students at participating schools were eligible to take part, and an invitation letter was sent to all parents/carers of these children. Anthropometric (including height and weight) and health-utility data were collected from children with parental consent for study participation. Measurements were undertaken in school by trained researchers using standardised protocols. BMI values were calculated using the standard BMI calculation (weight in $\mathrm{kg} /$ height in $\mathrm{m}^{2}$ ). The weight-status groups were then formulated according to the UK 1990 BMI cut-offs [12] as recommended by NICE, UK [13]. Baseline (BL) data were collected when the children were in school year 1 , aged 5-6 years. The intervention then ran for 1 year, and follow-up data were collected at 3 (F1), 18 (F2) and 30 (F3) months post intervention, with children aged 7-8, 8-9 and 9-10 years, respectively. Only half the schools were followed up at the final (30-month) follow-up point, as followup two was the primary end point of the trial [11].

The primary health-utility measure used was the Child Health Utility-9D (CHU-9D) instrument, designed for use within economic evaluations of interventions targeting paediatric populations [14]. The instrument was interviewer administered on a one-to-one basis. The CHU-9D consists of nine dimensions: worried; sad; annoyed; tired; pain; sleep; daily routine; schoolwork/homework and being able to join in activities, each of which has five severity levels. Each of the possible 1,953,125 health states described by the instrument can then be assigned a unique utility value ranging from 0.33 (worst health state) to 1 (best health state) using an algorithm that reflects the preference weight attached to each dimension [15]. These dimensions were originally identified through in-depth qualitative interviews with young people who had a variety of chronic and acute health problems to explore how their health affects their lives [14].

There are many potential methodological issues with measuring preference-based HRQL in children that has led to the validity of instruments being explored. Canaway and Frew [16] examined the performance of two GPBMs (the CHU-9D and the EQ-5D-Y [17]) in a pilot study for the WAVES trial, and found that although children aged 6-7 years could feasibly complete the instruments when administered by an interviewer, the reliability of the instruments was still uncertain. This pilot study concluded that 
the CHU-9D was the superior instrument, and it was recommended that the EQ-5D-Y should not be used to calculate utility values, until appropriate tariff values were available [16]. Frew et al. [5] contributed further evidence to the construct validity of the CHU-9D finding that HRQOL was associated with deprivation, and therefore the instrument was discriminating between these groups of children with known differences. Results from the same study also showed that the mean CHU9D values were significantly higher for children who reported individual PedsQL scores greater than the sample median PedsQL score [5]. Furthermore, Stevens [15] carried out a feasibility study and concluded that the CHU-9D could be used in the economic evaluation of paediatric interventions. For these reasons, the CHU9D was the instrument of choice for the WAVES study.

\section{Statistical analyses}

Three analyses were undertaken to explore the relationship between weight status and health-utility values over the study period.

First, to explore the relationship between health utility and weight status, descriptive statistics are reported. The paper reports the mean health-utility values (and SD) by weight status at baseline and follow-up points one, two and three as the children age.

Second, to examine the impact of weight status on the dimensions of health, the distributions of responses to each dimension of the CHU-9D were examined by weight status. For this analysis, weight status was divided into two groups- 'healthy/underweight' and 'overweight/obese'. The Chi-squared test was used to examine whether there were any differences between the patterns of responses in the two groups.

Finally, to model the relationship between health utility and weight status, a multi-level regression model, with random effects at the school level, was developed. The model controlled for potential confounders including gender, ethnicity, and deprivation, where deprivation was based on the IMD scores derived from the home postcode [11]. These co-variates were selected as prior research suggests they are associated with health-related quality of life [5, 18, 19]. It was hypothesised that higher utility scores would be observed in children of a healthy weight compared to those who are overweight or obese and that this relationship would become stronger as children age. There is some evidence that physical activity has an impact on HRQL in children, i.e. overweight children who are more active may have a greater HRQL than their peers who have a healthy weight but are less active [20]. We checked this by including a variable for exercise which used accelometery data. We ran the model with and without data for F3 to check whether missing data were an issue. The analysis was undertaken using Stata 13 [21].

\section{Results}

The baseline sample was 1397 children (mean age $=6$ years) of which $51 \%$ were male. The trial design necessitated that only half the cohort was followed up at the final time point, and there was also attrition throughout the study. Therefore, the participants measured at follow-up time points were $n(\mathrm{~F} 1)=1252, n(\mathrm{~F} 2)=1141$ and $n(\mathrm{~F} 3)=489$ with corresponding sample mean ages of, 8,9 and 10 years, respectively. The study population had a diverse ethnic and socioeconomic mixes with $55 \%$ being non-white and $55 \%$ from the most-deprived quintile. Participant characteristics are shown in Table 1.

Table 2 shows the distribution of CHU-9D dimensions by weight status at the three follow-up points. Overall the majority of the population had no or very few problems to report across all CHU-9D dimensions regardless of their weight status. The only dimension for which a statistically significant difference in the distribution of responses between the underweight/healthy weight and the overweight/ obese groups was indicated was for ability to join in activities ('Activities'), and this was only evident at the final follow-up (mean age of 10 years).

The results of the multi-level linear regression to examine the impact of weight status on health utility whilst controlling for potential confounders are shown in Table 3. There was no statistically significant difference in health-utility values between those in different weight status groups indicating that for the age groups considered, there is no association between health utility and weight status. The coefficient for age, however, was statistically significant $(0.02495 \%$ CI 0.022-0.027), and furthermore, deprivation was also significant with children in the most-deprived groups having a lower health utility than those in the least-deprived group. When F3 data were omitted from the model, the results were near identical (see supplementary materials Table 1).

When we included the variable for exercise, the coefficient was very small and non-significant, implying that exercise itself does not have a significant impact on children's HRQL (see supplementary materials Table 2).

\section{Discussion}

With childhood obesity projected to rise, there have been attempts to address this growing public health concern through the promotion of healthy lifestyles and through obesity prevention and management schemes. This study contributes to the growing body of research in childhood 
Table 1 Characteristics of trial participants

\begin{tabular}{|c|c|c|c|c|}
\hline Characteristics & Baseline & Follow-up 1 & Follow-up 2 & Follow-up 3 \\
\hline Age: Mean (SD) $(n=)$ & $6.2(0.307)(n=1397)$ & $7.6(0.298)(n=1252)$ & $8.9(0.302)(n=1141)$ & $9.6(0.306)(n=489)$ \\
\hline \multicolumn{5}{|l|}{ Weight status: $n(\%)$} \\
\hline Underweight & $40(2.87)$ & $37(2.96)$ & $32(2.81)$ & $8(1.67)$ \\
\hline Healthy weight & $1055(75.79)$ & $879(70.43)$ & $743(65.29)$ & $298(62.21)$ \\
\hline Overweight & $125(8.98)$ & $140(11.22)$ & $143(12.57)$ & $68(14.20)$ \\
\hline Obese & $172(12.36)$ & $192(15.38)$ & $220(19.33)$ & $105(21.92)$ \\
\hline CHU-9D: Mean (SD) $(n=)$ & $0.826(0.139)(n=1350)$ & $0.863(0.107)(n=1215)$ & $0.896(0.091)(n=1128)$ & $0.905(0.088)(n=485)$ \\
\hline Underweight & $0.855(0.131)(n=38)$ & $0.825(0.13)(n=37)$ & $0.898(0.1)(n=32)$ & $0.908(0.07)(n=8)$ \\
\hline Healthy weight & $0.826(0.14)(n=1022)$ & $0.863(0.11)(n=852)$ & $0.897(0.09)(n=729)$ & $0.904(0.09)(n=295)$ \\
\hline Overweight & $0.805(0.143)(n=118)$ & $0.866(0.1)(n=138)$ & $0.899(0.09)(n=140)$ & $0.911(0.09)(n=68)$ \\
\hline Obese & $0.829(0.128)(n=167)$ & $0.865(0.11)(n=182)$ & $0.89(0.1)(n=217)$ & $0.908(0.09)(n=104)$ \\
\hline \multicolumn{5}{|l|}{ Gender: $n(\%)(n=1467)$} \\
\hline Male & $749(51.06)$ & & & \\
\hline Female & $718(48.94)$ & & & \\
\hline \multicolumn{5}{|l|}{ Ethnicity: $n(\%)$} \\
\hline White & $658(45.38)$ & & & \\
\hline South Asian & $443(30.53)$ & & & \\
\hline Black African Caribbean & $115(7.93)$ & & & \\
\hline Other & $235(16.20)$ & & & \\
\hline \multicolumn{5}{|l|}{ Deprivation quintile: $n(\%)$} \\
\hline Most deprived & $803(55.53)$ & & & \\
\hline 2 & $261(18.05)$ & & & \\
\hline 3 & $164(11.34)$ & & & \\
\hline 4 & $122(8.44)$ & & & \\
\hline Least deprived & $96(6.64)$ & & & \\
\hline
\end{tabular}

obesity and the relationship between weight status and preference-based health utility over a period of time using the specially designed child utility instrument, the CHU-9D, within an ethnically and socioeconomically diverse population within the UK.

The main aim of this study was to investigate the longitudinal relationship between weight status and health utility in children during the middle-years phase. The results suggest there is no statistically significant relationship between weight status and health utility when measured using the CHU-9D during this time period. After adjusting for known potential confounders, for all ages, the relationship between health utility and weight status was negligible and not statistically significant. The study also found little evidence of differences in the distributions of response to the CHU9D dimensions by weight status as the children aged. One dimension (activities) at the final follow-up was significantly different between the two groups. Given the consistent lack of evidence for differences between the two groups with regard to all other dimensions at all other time points; the smaller sample size at this time point resulting in low numbers in several cells; and the use of multiple testing, this outcome should be interpreted with caution. These results support the findings of three studies [5-7] where no link was found, however, differing from the findings of two other papers $[8,9]$ which suggest an association.

As well as the main outcome of the effect of obesity on reported health utility as children grow older, another interesting finding emerged. Health utility was lower for children who were in the most-deprived quintile. This result indicates that the CHU-9D is capable of discriminating between subgroups of children based on deprivation.

One of the study's main strengths is its design. First, all height and weight measurements were undertaken by trained researchers using standardised equipment and operating procedures to ensure consistency in measurement. Similarly, the CHU-9D was interviewer administered to ensure that the children engaged with the task. Unlike previous studies of the effect of weight status on health utility, this study included a longitudinal element which allowed for an examination of how that relationship changed during the middle years.

The main weakness of the study surrounds the difficulty in deciphering whether there is truly no relationship between weight status and health utility as children grow older from 6 to 10 years, or whether the CHU-9D is simply 


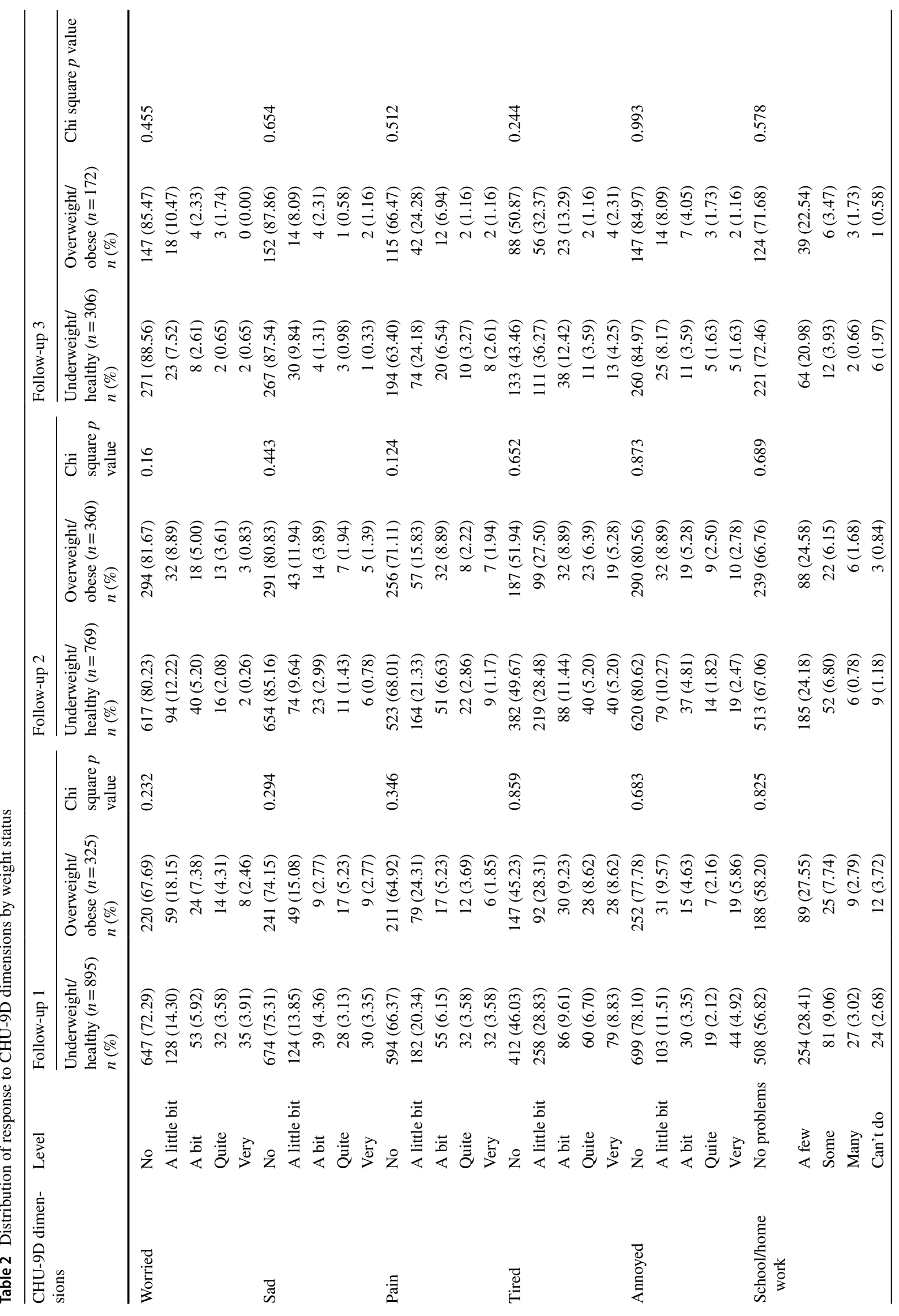




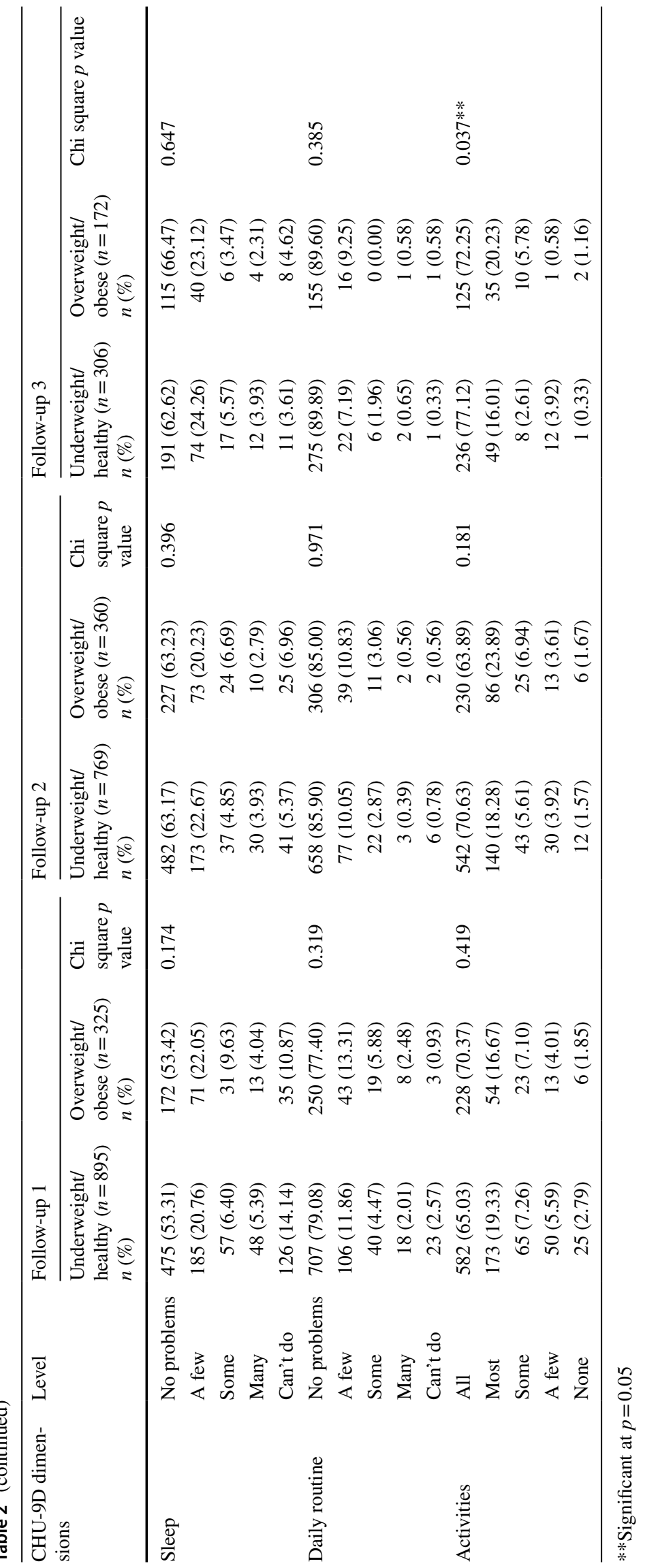


Table 3 Regression results from analyses investigating the impact of weight status on health utility

\begin{tabular}{lllll}
\hline CHU-9D score & Coef. & $p$ value & \multicolumn{2}{c}{$95 \%$ Conf. Interval } \\
\cline { 3 - 5 } & & & Lower CI & Upper CI \\
\hline Age & 0.024292 & $0.000^{* *}$ & 0.021559 & 0.027024 \\
Gender $^{\mathrm{a}}$ & & & & \\
Female & 0.001701 & 0.634 & -0.00529 & 0.008692 \\
Weight status & & & & \\
Healthy weight & 0.000437 & 0.968 & -0.02068 & 0.021551 \\
Overweight & -0.00126 & 0.915 & -0.02438 & 0.021864 \\
Obese & 0.003166 & 0.782 & -0.01924 & 0.025576 \\
Ethnicity & & & & \\
Asian & -0.00174 & 0.739 & -0.01196 & 0.008478 \\
African Caribbean & -0.00843 & 0.272 & -0.02346 & 0.006601 \\
Other & -0.00159 & 0.782 & -0.01284 & 0.009661 \\
Not known & 0.002852 & 0.936 & -0.06716 & 0.072859 \\
IMD quintile & & & & \\
2 & & & -0.00062 & 0.021312 \\
3 & 0.010348 & 0.064 & -0.0023 & 0.023146 \\
4 & 0.010426 & 0.108 & -0.01386 & 0.016043 \\
Least deprived & 0.029459 & $0.001 * *$ & 0.011988 & 0.04693 \\
cons & 0.670001 & $0 * *$ & 0.638859 & 0.701143 \\
\hline
\end{tabular}

Reference categories: ${ }^{\mathrm{a}}$ Male, ${ }^{\mathrm{b}}$ Underweight, ${ }^{\mathrm{c}}$ White, ${ }^{\mathrm{d}}$ Most deprived

**Significant at $p=0.05$

not sensitive enough to identify one. The CHU-9D was originally designed for use with children of 7-11 year olds [22], and although Canaway and Frew [16] found the instrument to be acceptable and feasible for children aged 6-7 years, there are still concerns regarding the instrument's reliability $[16,23]$ in younger children. There exists evidence on the relationship between weight status and HRQL in the non-preference HRQL literature. Ottova et al. [24] used data from the KIDSCREEN health interview survey [25] which included over 17,000 children and adolescents aged 8-18 years from 10 European countries. Results of this study support the hypothesis that overweight or obese children and adolescents have a lower HRQL than their healthyweight counterparts. And, these results are consistent with other HRQL studies [26-29] that used non-preference-based instruments and found evidence that paediatric obesity impacts on self-esteem and quality of life.

The finding that HRQL increases systematically with children getting older is interesting, and even though the analysis included known potential confounders, it is possible that there were other unknown factors that were omitted.

This result has implications for the economic evaluation of childhood obesity interventions. One could argue that having no negative effect identified by preference-based measures is not necessarily a concern for economic evaluation as long-term modelling that extrapolates results into the future could account for future cost-savings and benefit from preventing obesity into adulthood. Whilst this might be true, the reality is that the clinical and public health evidence base is strongly in favour of preventing childhood obesity, and if the conventional application of measuring QALYs is pursued in individuals in the middle-years phase of childhood, then any gains from intervening are not going to be realised in QALY terms. As a result, conventional rules for judging whether an intervention is deemed cost-effective using a cost/QALY ratio may disadvantage interventions targeting childhood obesity.

Funding This study was funded by the National Institute for Health Research (NIHR) Health Technology Assessment Programme (Number: $06 / 85 / 11)$.

\section{Compliance with ethical standards}

Conflict of interest The authors declare that they have no conflict of interest.

Ethical approval All procedures performed in studies involving human participants were in accordance with the ethical standards of the institutional and/or national research committee and with the 1964 Helsinki declaration and its later amendments or comparable ethical standards. NHS research ethics committee number: 10/H1202/69.

Informed consent Informed consent was obtained from all individual participants included in the study.

Open Access This article is distributed under the terms of the Creative Commons Attribution 4.0 International License (http://creativeco mmons.org/licenses/by/4.0/), which permits unrestricted use, distribution, and reproduction in any medium, provided you give appropriate credit to the original author(s) and the source, provide a link to the Creative Commons license, and indicate if changes were made.

\section{References}

1. Drummond, M. F., Sculpher, M. J., Claxton, K., Stoddart, G. L., \& Torrance, G. W. (2015). Methods for the economic evaluation of health care programmes. Oxford: OUP.

2. Feeny, D. (2000). A utility approach to the assessment of healthrelated quality of life. Medical Care, 38, II151-II154.

3. Whitehead, S. J., \& Ali, S. (2010). Health outcomes in economic evaluation: The QALY and utilities. British Medical Bulletin, 96, $5-21$.

4. Waters, E., de Silva-Sanigorski, A., Burford, B. J., Brown, T., Campbell, K. J., Gao, Y., Armstrong, R., Prosser, L., \& Summerbell, C. D. (2011). Interventions for preventing obesity in children. Cochrane Database of Systematic Reviews. https://doi. org/10.1002/14651858.CD001871.pub3.

5. Frew, E. J., Pallan, M., Lancashire, E., Hemming, K., \& Adab, P. (2015). Is utility-based quality of life associated with overweight in children? Evidence from the UK WAVES randomised controlled study. BMC Pediatrics, 15, 211. 
6. Canaway, A. G., \& Frew, E. J. (2014). Is utility-based quality of life in children aged 6-7 years affected by Body Mass Index (BMI)? International Journal of Obesity, 38(8), 1146.

7. Belfort, M. B., Zupancic, J. A., Riera, K. M., Turner, J. H., \& Prosser, L. A. (2011). Health state preferences associated with weight status in children and adolescents. BMC Pediatrics, 11, 12.

8. Chen, G., Ratcliffe, J., Olds, T., Magarey, A., Jones, M., \& Leslie, E. (2014). BMI, health behaviors, and quality of life in children and adolescents: A school-based study. Pediatrics, 133, e868-e874.

9. Boyle, S. E., Jones, G. L., \& Walters, S. J. (2010). Physical activity, quality of life, weight status and diet in adolescents. Quality of Life Research, 19, 943-954.

10. Guhn, M., Janus, M., \& Hertzman, C. (2007). The early development instrument: Translating school readiness assessment into community actions and policy planning. Early Education and Development, 18, 369-374.

11. Adab, P., Pallan, M. J., Lancashire, E. R., Hemming, K., Frew, E., Griffin, T., Barrett, T., Bhopal, R., Cade, J. E., Daley, A., Deeks, J., Duda, J., Ekelund, U., Gill, P., Mcgee, E., Parry, J., Passmore, S., \& Cheng, K. K. (2015). A cluster-randomised controlled trial to assess the effectiveness and cost-effectiveness of a childhood obesity prevention programme delivered through schools, targeting 6-7 year old children: The WAVES study protocol. BMC Public Health, 15, 488.

12. Cole, T. J., Freeman, J. V., \& Preece, M. A. (1995). Body mass index reference curves for the UK, 1990. Archives of Disease in Childhood, 73, 25-29.

13. NICE. (2006). Obesity guidance on the prevention, identification, assessment and management of overweight and obesity in adults and children. Retrieved from http://www.nice.org.uk/CG43.

14. Stevens, K. (2009). Developing a descriptive system for a new preference-based measure of health-related quality of life for children. Quality of Life Research, 18, 1105-1113.

15. Stevens, K. (2012). Valuation of the child health utility 9D index. PharmacoEconomics, 30, 729-747.

16. Canaway, A., \& Frew, E. (2013). Measuring preference-based quality of life in children aged 6-7 years: A comparison of the performance of the CHU-9D and EQ-5D-Y - The WAVES Pilot Study. Quality of Life Research, 22(1), 173-183.

17. Wille, N., Badia, X., Bonsel, G., Burström, K., Cavrini, G., Devlin, N., Egmar, A.-C., Greiner, W., Gusi, N., Herdman, M., Jelsma, J., Kind, P., Scalone, L., \& Ravens-Sieberer, U. (2010). Development of the EQ-5D-Y: A child-friendly version of the EQ-5D. Quality of Life Research, 19, 875-886.
18. Minet Kinge, J., \& Morris, S. (2010). Socioeconomic variation in the impact of obesity on health-related quality of life. Social Science \& Medicine, 71(10), 1864-1871.

19. Mcdonough, C., Dunkley, A. J., Aujla, N., Morris, D., Davies, M. J., \& Khunti, K. (2013). The association between body mass index and health-related quality of life: Influence of ethnicity on this relationship. Diabetes, Obesity and Metabolism, 15(4), 342-348.

20. Wafa, S. W. W. B. S. S. T., Shahril, M. R. B., Ahmad, A. B., Zainuddin, L. R. B., Ismail, K. F. B., Aung, M., \& M. T. \& Mohd Yusoff, N. A. B. (2016). Association between physical activity and health-related quality of life in children: A cross-sectional study. Health and Quality of Life Outcomes, 14, 71.

21. StataCorp. (2013). Stata statistical software: Release 13. College Station, TX: StataCorp LP.

22. Stevens, K. (2011). Assessing the performance of a new generic measure of health-related quality of life for children and refining it for use in health state valuation. Applied Health Economics and Health Policy, 9, 157-169.

23. Furber, G., \& Segal, L. (2015). The validity of the Child Health Utility instrument (CHU9D) as a routine outcome measure for use in child and adolescent mental health services. Health and Quality of Life Outcomes, 13, 22.

24. Ottova, V., Erhart, M., Rajmil, L., Dettenborn-Betz, L., \& RavensSieberer, U. (2012). Overweight and its impact on the healthrelated quality of life in children and adolescents: Results from the European KIDSCREEN survey. Quality of Life Research, 21, 59-69.

25. Ravens-Sieberer, U. \& the European KIDSCREEN Group. (2006). The KIDSCREEN questionnaires-Quality of life questionnaires for children and adolescents_Handbook. Lengerich: Pabst Science Publisher.

26. Schwimmer, J. B., Burwinkle, T. M., \& Varni, J. W. (2003). Health-related quality of life of severely obese children and adolescents. JAMA, 289, 1813-1819.

27. Williams, J., Wake, M., Hesketh, K., Maher, E., \& Waters, E. (2005). Health-related quality of life of overweight and obese children. JAMA, 293, 70-76.

28. Wille, N., Bullinger, M., Holl, R., Hoffmeister, U., Mann, R., Goldapp, C., Reinehr, T., Westenhöfer, J., Egmond-Froehlich, A., \& Ravens-Sieberer, U. (2010). Health-related quality of life in overweight and obese youths: Results of a multicenter study. Health and Quality of Life Outcomes, 8, 36.

29. Griffiths, L. J., Parsons, T. J., \& Hill, A. J. (2010). Self-esteem and quality of life in obese children and adolescents: A systematic review. International Journal of Pediatric Obesity, 5, 282-304. 